\title{
An Active Shielding Control Method for a Wireless Power Transfer System under Misalignment Conditions
}

\author{
Jangyong Ahn · Jedok Kim · Dongryul Park $\cdot$ Haerim Kim · Seungyoung Ahn*
}

\begin{abstract}
This paper proposes a control method for an active shield system using an independent power source under misalignment conditions. The proposed method controls the current strength and phase of the shielding system according to magnetic field leakage characteristics with high shielding performance (SP) under misalignment conditions. The current strength and phase of the shielding system were determined by the current strength and mutual inductance of the wireless power transfer (WPT) and shielding coils. Additionally, we considered $x$ and $y$ misalignment conditions and analyzed SP and power transfer efficiency (PTE). The proposed method was verified through simulations and experiments in terms of SP and PTE. The experiments confirmed that, due to the shielding system control, SP increased from 49.22\% to $61.74 \%$ under misalignment conditions, but there was no additional PTE reduction due to shielding coil control under these conditions.
\end{abstract}

Key Words: Active Shield, Electromagnetic Field, Misalignment, Wireless Power Transfer.

\section{INTRODUCTION}

Currently, wireless power transfer (WPT) technology is being studied and used for various applications, including low-power mobile phones and high-power wireless charging systems, such as those used for drones and electric vehicles (EVs) [1]. Since this technology can solve the problems of limited battery storage, it is expected to be useful for various purposes in the future.

However, WPT systems cause electromagnetic field (EMF) leakage in their vicinities. This leakage can adversely affect nearby human bodies; therefore, to ensure the minimum safe human exposure to EMF leakage from such systems, international guidelines and standards recommend that the leakage should meet reference levels [2-4].

To reduce EMF leakage from WPT systems, different types of shielding methods have been investigated, which can be divided into passive, reactive, and active shielding categories [5]. Passive shielding methods use metal or magnetic materials to guide or block the leakage of magnetic fields, but metal shielding results in a significant reduction in power transfer efficiency (PTE). Also, magnetic materials have low shielding performance (SP). For reactive shielding methods, transmitting (TX) and receiving $(\mathrm{RX})$ coils cause induced current to flow through the shielding $(\mathrm{SH})$ coil to generate a counteracting magnetic field. Lastly, active shielding methods intentionally generate canceling EMFs with the desired magnitude and phase. Under these

Manuscript received December 22, 2020 ; Revised May 5, 2021 ; Accepted June 11, 2021. (ID No. 20201222-209J)

The Cho Chun Shik Graduate School of Green Transportation, Korea Advanced Institute of Science and Technology (KAIST), Daejeon, Korea.

"Corresponding Author: Seungyoung Ahn (e-mail: sahn@kaist.ac.kr)

This is an Open-Access article distributed under the terms of the Creative Commons Attribution Non-Commercial License (http://creativecommons.org/licenses/by-nc/4.0) which permits unrestricted non-commercial use, distribution, and reproduction in any medium, provided the original work is properly cited.

(c) Copyright The Korean Institute of Electromagnetic Engineering and Science. 
operating conditions, active shielding methods have a higher SP than reactive shielding methods [6].

Active shielding systems can be divided into two types: one using a dependent power source and the other using an independent power source [6-8]. The active shielding method using a dependent power source generates a canceling magnetic field because the TX and RX coil windings turn in opposite directions. Opposite-direction winding has negative mutual inductance for forward-direction winding, so the effective inductances of the TX and RX coils decrease. This can be a drawback that significantly reduces the PTE of the system. Also, the PTE decreases when the opposite-direction winding increases for high SP. To overcome this limitation, studies have proposed an active shielding system using an independent power source $[6$, 8]. The active shielding system operates independently of the WPT system; thus, it can achieve high SP by controlling the current strength and phase of the $\mathrm{SH}$ coil.

The active shielding methods in previous works have been studied for high SP in an aligned state, but they have not considered misalignment conditions. A WPT system may not always transmit power in perfect alignment due to the movement of the transceiver [9-11]. In this case, the SP decreases due to changes in the magnetic field generation characteristics. Consequently, human exposure to EMFs can increase due to decreased SP under misalignment conditions [12], necessitating the development of an active shielding control method that uses an independent power source under misalignment conditions.

This paper proposes a control method for an active shielding system using an independent power source under misalignment conditions. The active shielding system is controlled by reflecting the current strength and phase of the WPT system under misalignment conditions, and the proposed method was analyzed and verified using simulations and experiments. The organization of this paper is as follows: Section II presents the misalignment condition analysis, Section III explains the verification based on the simulations and experiments, and Section IV presents the conclusions.

\section{MisAlignMENT CONDITION ANALYSIS}

\section{Mutual Inductance Analysis}

Fig. 1 shows the geometry of the TX, RX, and SH coils under misalignment conditions. I, $\phi$, and $r$ refer to the current, phase, and radius of the coil, respectively. A misalignment condition is set for $\mathrm{RX}$ and $\mathrm{SH}$ coils, which moves in the $x$ and $y$ directions, increasing the distance from the TX coil. The RX and SH coils are located at a position moved by $a$ and $b$ on the $x$ and $y$ axes of the Cartesian coordinate. $d_{p-T X}, d_{p-R X}, d_{p-S H}$ are the distances from the center of the TX, RX, and SH coils to point $p\left(x_{p}, y_{p}, z_{p}\right)$, calculated by Eqs. (1) and (2). The angles between the $z$ axes are

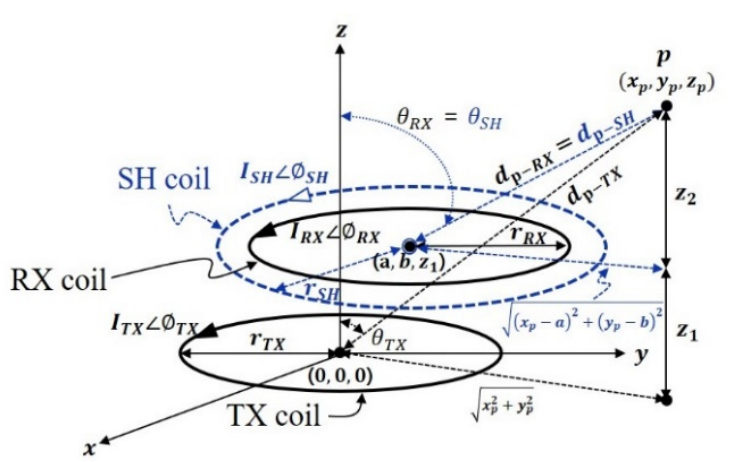

Fig. 1. Geometry of TX, RX, and SH coils.

expressed as $\theta_{T X}, \theta_{R X}$, and $\theta_{S H}$. The magnetic field strength from each coil at point $p$ can be calculated by Eq. (3), where $\vartheta=\sin \theta \cos \varphi \cos \theta[6]$.

$$
\begin{gathered}
d_{p-T X}=\sqrt{\left(\sqrt{x_{p}^{2}+y_{p}^{2}}\right)^{2}+\left(z_{1}+z_{2}\right)^{2}} \\
d_{p-R X(S H)}=\sqrt{\left(\sqrt{\left(x_{p}-a\right)^{2}+\left(y_{p}-b\right)^{2}}\right)^{2}+z_{2}^{2}} \\
B_{p-\text { coil }}=j \mu_{o} \frac{k r^{2} I_{0} \cos \theta}{2 d^{2}}\left[1+\frac{1}{j k d}\right] e^{-j k d} \cdot \vartheta \\
-\mu_{o} \frac{(k r)^{2} I_{0} \sin \theta}{4 d}\left[1+\frac{1}{j k d}-\frac{1}{(k r)^{2}}\right] e^{-j k d} \cdot \vartheta
\end{gathered}
$$

Fig. 2 shows the mutual inductance $(M)$ variations of the RX and SH coils from the TX coil due to misalignments. $M_{T X R X}$ is

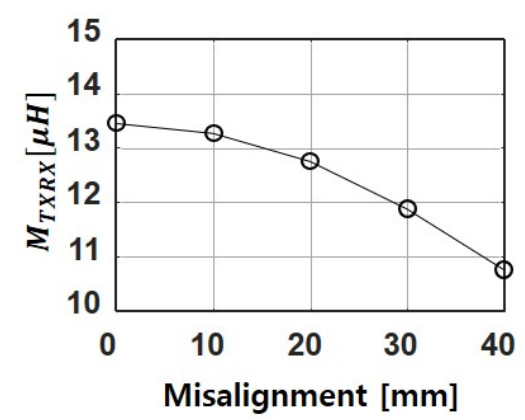

(a)

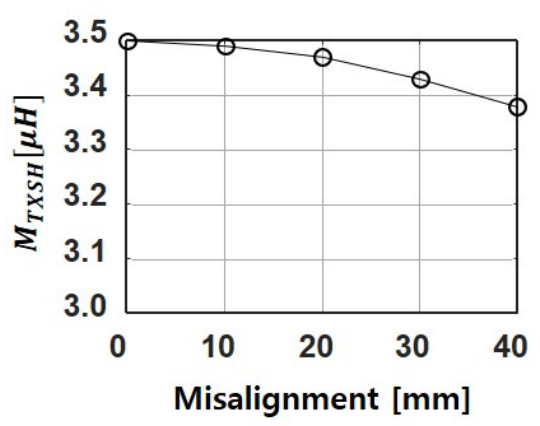

(b)

Fig. 2. Mutual variations according to the misalignment of the $\mathrm{RX}$ and SH coils: (a) $M_{T X R X}$ and (b) $M_{T X S H}$. 
the $M$ between the TX and RX coils, and $M_{T X S H}$ is the $M$ between the TX and SH coils. The TX and RX coils have identical radii of $95 \mathrm{~mm}$, and the $\mathrm{SH}$ coil radius is $150 \mathrm{~mm}$. The air gap between the TX and RX coils is $75 \mathrm{~mm}$. The displacement of the RX and SH coils ranges from 0 to $40 \mathrm{~mm}$ in $10 \mathrm{~mm}$ steps on the $x$ axis.

Although the $\mathrm{RX}$ and $\mathrm{SH}$ coils have identical displacements, the variations of $M_{T X R X}$ and $M_{T X S H}$ differ because of the radius difference between the RX and SH coils.

$M$ is determined by geometry, including the radius and distance of each coil. $M$ between two coils is calculated using the Neumann formula according to Eq. (4) [13]. $g$ is a distance between two coils, and $M$ can be expressed in terms of the coupling coefficient $(k)$ and the inductances of two coils determined by Eq. (5).

$$
\begin{gathered}
M=\frac{\mu_{0}}{4 \pi} \int_{0}^{2 \pi} \int_{0}^{2 \pi} \frac{r^{2} \cos \left(\theta_{1}-\theta_{2}\right)}{\sqrt{2 r^{2}+g^{2}-2 r^{2} \cos \left(\theta_{1}-\theta_{2}\right)}} \mathrm{d} \theta_{1} \mathrm{~d} \theta_{2}(H) \\
M=k \sqrt{L_{1} L_{2}}
\end{gathered}
$$

$M$ between two coils decreases as misalignment occurs in the WPT system. In other words, the operation characteristics of the WPT system change under misalignment conditions due to $M$ variations; therefore, this $M$ variation should be considered to control the active shield system for high SP.

\section{Power Transfer Efficiency Analysis}

Fig. 3 shows the equivalent circuit of the WPT system, including the independent active shielding system. $V_{I N}$ and $V_{S H}$ are the voltage sources of the TX and SH system; $S_{1}-S_{8}$ are the MOSFETs for full-bridge inverters of TX and SH; and $I_{T X}, I_{R X}$, and $I_{S H}$ are the currents of the TX, RX, and SH coils.

The TX and RX systems comprise inductance $L$, capacitance $C$, and resistance $R$, respectively; $R_{L}$ is the load resistance. The $\mathrm{SH}$ system is composed of inductance and resistance without a resonant capacitor.

Based on Kirchhoff's voltage law (KVL), the impedance of the circuit can be expressed by Eqs. (6)-(8). The impedance changes according to KVL because the $M$ changes under misa-

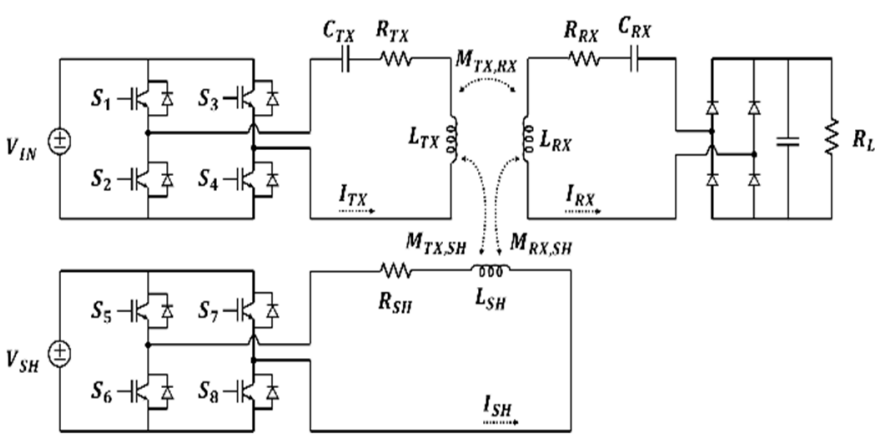

Fig. 3. Equivalent circuit of a WPT system with an active shielding system using an independent power source. lignment conditions; consequently, the voltage and current characteristics can also vary.

$$
\begin{gathered}
Z_{T X}=R_{T X}+j \omega L_{T X}+\frac{1}{j \omega C_{T X}}+j \omega M_{T X R X}\left(\frac{I_{R X}}{I_{T X}}\right)+j \omega M_{T X S H}\left(\frac{I_{S H}}{I_{T X}}\right), \\
0=R_{R X}+j \omega L_{R X}+\frac{1}{j \omega C_{R X}}+j \omega M_{T X R X}\left(\frac{I_{T X}}{I_{R X}}\right)+j \omega M_{R X S H}\left(\frac{I_{S H}}{I_{R X}}\right), \\
Z_{S H}=R_{S H}+j \omega L_{S H}+j \omega M_{T X S H}\left(\frac{I_{T X}}{I_{S H}}\right)+j \omega M_{R X S H}\left(\frac{I_{R X}}{I_{S H}}\right) .
\end{gathered}
$$

Fig. 4 shows the current strengths and PTE variations according to $k$ under identical load power $\left(P_{\text {Load }}\right)$. The TX and RX coils have identical $150 \mathrm{~mm}$ radii, and the current strength of the TX coil is calculated while changing $k$ from 0.05 to 0.3 .

Fig. 4(a) shows the variation in $I_{T X}$ according to the $k$ variation. The more $k$ decreases, the more $I_{T X}$ increases, meaning that power loss increases in terms of ohm loss $I^{2} R$ under misalignment conditions. Furthermore, the required $I_{S H}$ should be increased for high SP because of the increased $I_{T X}$, resulting in increased power loss in the $\mathrm{SH}$ system.

Fig. 4(b) shows the variations in the PTE of the system according to $k$ variation. The decrease in $k$ results in a decrease in the PTE. Decreasing $k$ due to misalignment reduces the im-

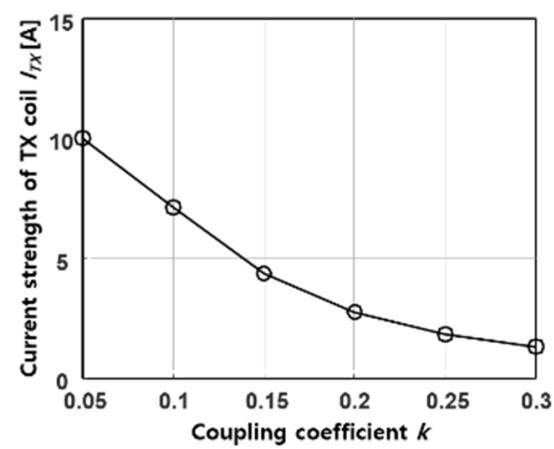

(a)

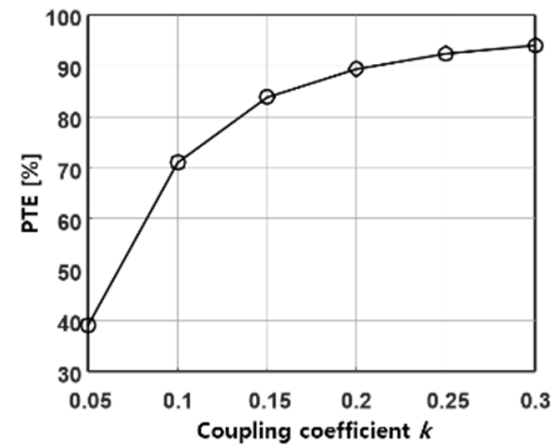

(b)

Fig. 4. Variation in the WPT system characteristics according to the coupling coefficient: (a) TX coil current strength and (b) PTE of the system. 
pedance of the circuit and changes the characteristics of the system to low voltage and high current. The high current characteristic has the effect of increasing the power loss at the coil.

Also, when the SH coil is applied to the WPT system, $M_{T X S H}$ and $M_{R X S H}$ should be considered. The magnetic field of the $\mathrm{SH}$ coil is out of phase with that of the TX and RX coils, which causes a decrease in the effective inductance of the TX and RX coils, as shown by Eq. (9). Consequently, the PTE decreases when applying the SH system. The PTE of the WPT system, including the active shielding system using an independent power source, can be calculated using Eq. (10).

$$
\begin{gathered}
L_{\text {effective }}=L_{\text {self }}-M \\
\operatorname{PTE}(\%)=\frac{R_{L}\left|I_{R X}\right|^{2}}{R_{T X}\left|I_{T X}\right|^{2}+\left(R_{R X}+R_{L}\right)\left|I_{R X}\right|^{2}+R_{S H}\left|I_{S H}\right|^{2}} \times 100
\end{gathered}
$$

\section{Current and Phase Control of the Shielding System}

Fig. 5 shows the front view of the WPT system, including the $\mathrm{SH}$ coil. To achieve high SP, the current applied to the $\mathrm{SH}$ coil should be determined, considering misalignment conditions. However, since the positions and designs of the three coils of TX, RX, and SH differ, the composite magnetic field in space varies depending on the positions, meaning that the shielding current and phase of the shielding coil depend on the target point $p$.

Fig. 6(a) shows a magnetic vector diagram of the TX, RX, and $\mathrm{SH}$ coils in the alignment condition. The magnitude and phase of $B_{W P T}$ is calculated by Eqs. (11) and (12) [6]. The maximum SP is obtained when the SH coil has an identical magnetic field strength and $180^{\circ}$ of phase difference for the $B_{W P T}$; however, the magnetic field strengths of the TX and RX coils change under misalignment conditions.

$$
\begin{gathered}
B_{p-W P T}=\left(B_{p-T X}+B_{p-R X}\right) \cos \left(\frac{\emptyset_{R X}}{2}\right) \\
\emptyset_{p-W P T}=\tan ^{-1}\left(\frac{B_{p-R X}}{B_{p-T X}}\right)-\frac{90-\emptyset_{R X}}{2}
\end{gathered}
$$

Fig. 6(b)-6(d) show the magnetic flux density and phase changes according to the measurement point $p$ under misalign-

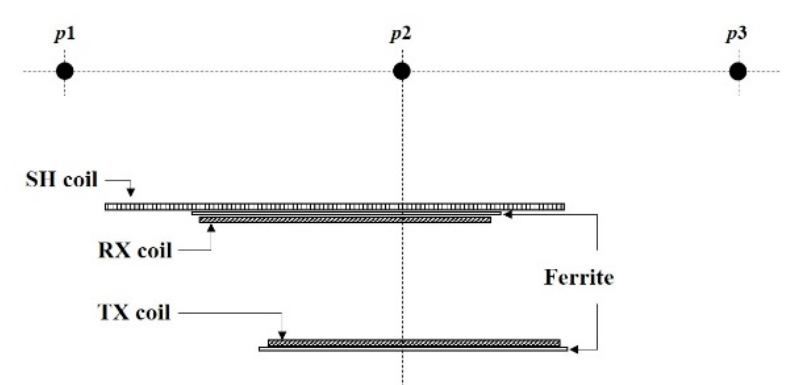

Fig. 5. Front view of the system under misalignment conditions for target point $p$.

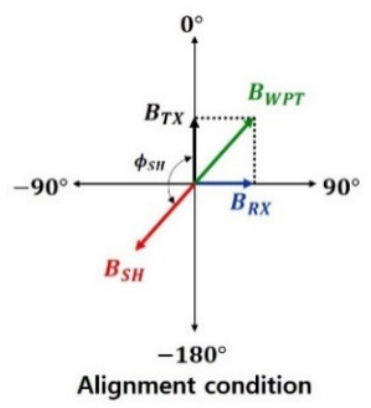

(a)

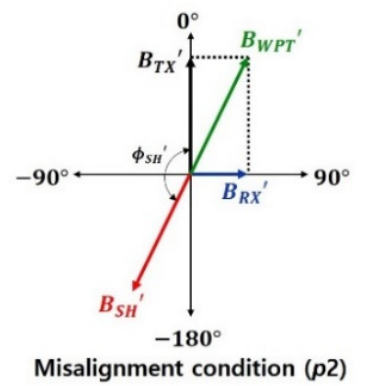

(c)

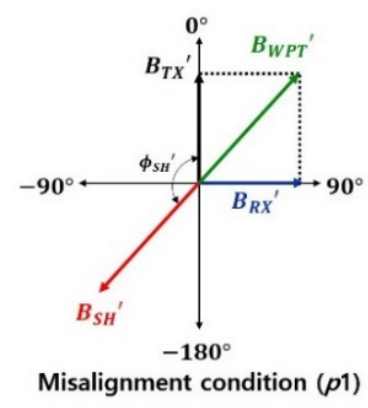

(b)

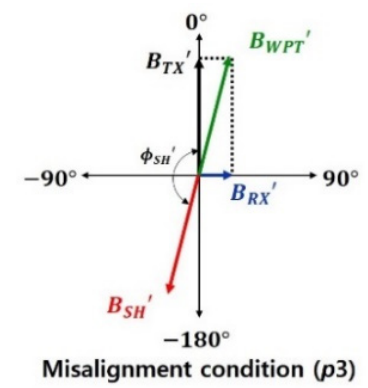

(d)
Fig. 6. Phasor diagram of (a) alignment and misalignment conditions at (b) $p 1,(\mathrm{c}) p 2$, and (d) $p 3$.

ment conditions. $B_{T X}{ }^{\prime}, B_{R X}{ }^{\prime}$, and $B_{W P T}{ }^{\prime}$ are the changed magnetic flux densities under misalignment conditions. The $B_{T X}{ }^{\prime}$ defined in Eq. (3) increases according to increased $I_{T X}{ }^{\prime}$, which is the TX coil current under misalignment conditions. $I_{T X}$ ' increases by decreasing $M_{T X R X}$ under misalignment conditions, as described in Section II-2. $B_{R X}{ }^{\prime}$ differs significantly depending on $p$ because, although $I_{R X}{ }^{\prime}$ is identical to $I_{R X}$, the distance to point $p$ differs. Compared to the alignment condition, $B_{R X}{ }^{\prime}$ increases at $p 1$, is equal at $p 2$, and decreases at $p 3$.

According to Eq. (12), the changes in $B_{p-T X}$ and $B_{p-R X}$ cause a change in $\Phi_{p-P W T}$. As the change occurs from $p 1$ to $p 3$, the distance from the RX coil increases; thus, $B_{p-R X}$ decreases and $B_{p-T X}$ becomes dominant. In this case, the $\phi_{p-S H}$ approaches the opposite direction of $\phi_{p-T X}$ as it goes from Fig. 6(b) to 6(d). Therefore, $B_{S H}{ }^{\prime}$ must be controlled according to the target point $p$ and $B_{W P T}{ }^{\prime}$ to achieve high SP.

Assuming that the RX and $\mathrm{SH}$ coils are located at a point (a, b, $\mathrm{z}_{1}$ ), as shown in Fig. 1, the distance between the TX and SH coils, $g^{\prime}$, is calculated using Eq. (13). $M_{T X R X}{ }^{\prime}$ can then be calculated using Eq. (4) by substituting the distance $g$ to $g^{\prime}$. Since an identical $P_{\text {Load }}$ is assumed, $V_{R X}{ }^{\prime}$ and $I_{R X}{ }^{\prime}$ are identical under the alignment condition, and $I_{T X}{ }^{\prime}$ can be calculated through $M_{T X R X}$ according to Eq. (14). Thus, using the calculated $I_{T X}{ }^{\prime}$ and the known $I_{R X}{ }^{\prime}$, the magnetic field strengths $B_{T X}{ }^{\prime}$ and $B_{R X}{ }^{\prime}$ at point $p$ can be calculated using Eq. (3). As a result, the magnitude and phase of the $I_{S H}{ }^{\prime}$ is determined based on the $B_{S H}{ }^{\prime}$ according to Eqs. (11) and (12). 


$$
\begin{gathered}
g^{\prime}=\sqrt{\left(a^{2}+b^{2}\right)^{2}+z_{1}^{2}} \\
I_{T X}{ }^{\prime}=\frac{j I_{R X^{\prime \prime}}\left(R_{R X}+j \omega L_{R X^{+}}+\frac{1}{j \omega C_{R X}}\right)}{\omega M_{T X R X^{\prime}}}
\end{gathered}
$$

\section{SIMULATION AND EXPERIMENTS}

\section{Simulation and Experimental Setup}

Fig. 7 shows the simulation and experimental model, and Fig. 8 shows the experimental setup. Also, Table 1 shows the electrical parameters of the WPT and SH coils, and $P_{\text {Load }}$ and PTE before and after applying the SH coil. The TX and RX coils had outer and inner radii of $95 \mathrm{~mm}$ and $35 \mathrm{~mm}$, respectively; the diameter of the wire was $4 \mathrm{~mm}$. Ferrite plates with widths and lengths of $200 \mathrm{~mm} \times 200 \mathrm{~mm}$ and heights of $4 \mathrm{~mm}$ were used for the TX and RX coils. The radius of the SH coil $r_{S H}$ was

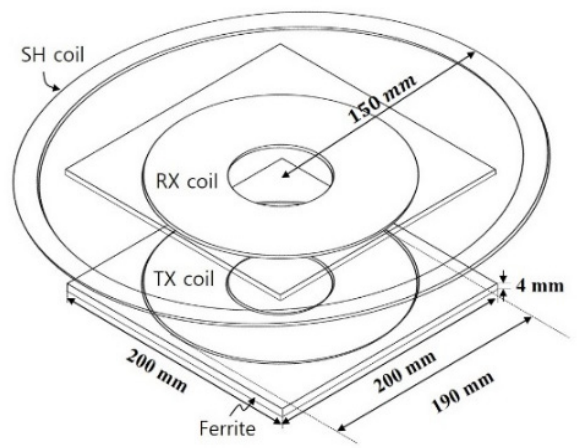

Fig. 7. Structures of the WPT system with a shielding system.

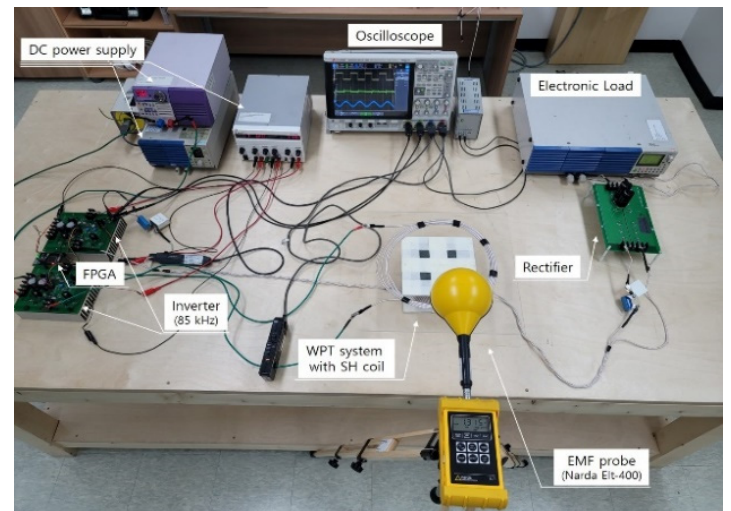

Fig. 8. Experimental setup.

Table 1. Specifications of the WPT system under alignment conditions at $85 \mathrm{kHz}$

\begin{tabular}{ccccccc}
\hline Coil & $\begin{array}{c}\text { Radius } \\
(\mathrm{mm})\end{array}$ & $\begin{array}{c}\text { \# of } \\
\text { turns }\end{array}$ & $\begin{array}{c}\mathrm{L} \\
(\mu H)\end{array}$ & $\begin{array}{c}\mathrm{C} \\
(n F)\end{array}$ & $\begin{array}{c}P_{\text {Load }} \\
(W)\end{array}$ & $\begin{array}{c}\text { PTE } \\
(\%)\end{array}$ \\
\hline $\mathrm{TX}$ & 95 & 15 & 54.75 & 68.99 & & \\
$\mathrm{RX}$ & 95 & 15 & 55.71 & 69.42 & 105.8 & 90.16 \\
$\mathrm{SH}$ & 150 & 5 & 15.85 & - & 112.7 & 88.53 \\
\hline
\end{tabular}

$150 \mathrm{~mm}$, and the number of turns was set to five. The operating frequency of the WPT system was $85 \mathrm{kHz}$, and $R_{L}$ was $10.016 \Omega$. Also, the input voltage was adjusted to an identical $P_{\text {Load }}$ of about $100 \mathrm{~W}$ for all measurements.

Fig. 9 shows the alignment and misalignment conditions considered in the simulations and experiments: alignment, which is the condition in which the RX and SH coils are aligned on the $\mathrm{z}$ axis with the TX coil; and misalignment, meaning that the $\mathrm{RX}$ and SH coils are misaligned by $40 \mathrm{~mm}$ in both the $x$ - and $y$-axis directions.

Fig. 10 shows the measurement points (MPs) used to analyze SP. The MPs were set as the center and periphery of the system from MP1 to MP9 at distances of $15 \mathrm{~cm}$ above the RX coil.

The results according to the alignment and misalignment conditions were as follows: as misalignment occurred, $I_{T X}$ increased. Regardless of the alignment condition, the $P_{\text {Load }}$ was identical, so the $I_{R X}$ did not change. However, when controlling for the misalignment condition, the $I_{S H}$ increased, and the $\Phi_{S H}$ shifted close to the opposite direction of the TX, which was closer to $-180^{\circ}$.

Table 2 shows the current strengths and phases of the TX, $\mathrm{RX}$, and $\mathrm{SH}$ coils under alignment and misalignment conditions. For the misalignment conditions, the current strengths and phases were controlled. The shielding system control set the

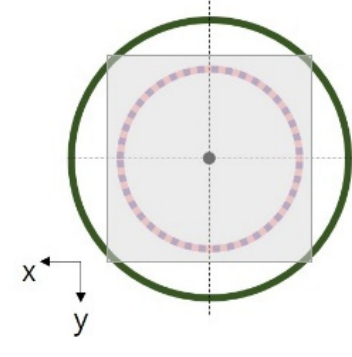

(a)

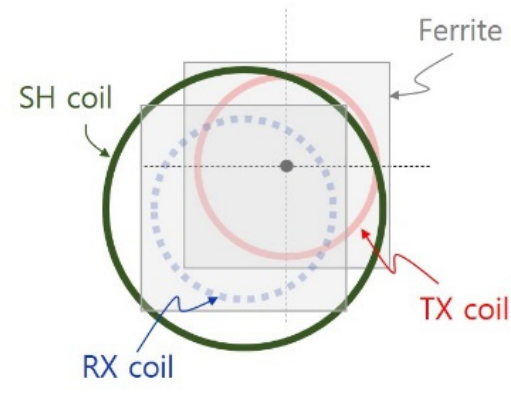

(b)
Fig. 9. Alignment conditions: (a) alignment and (b) misalignment.

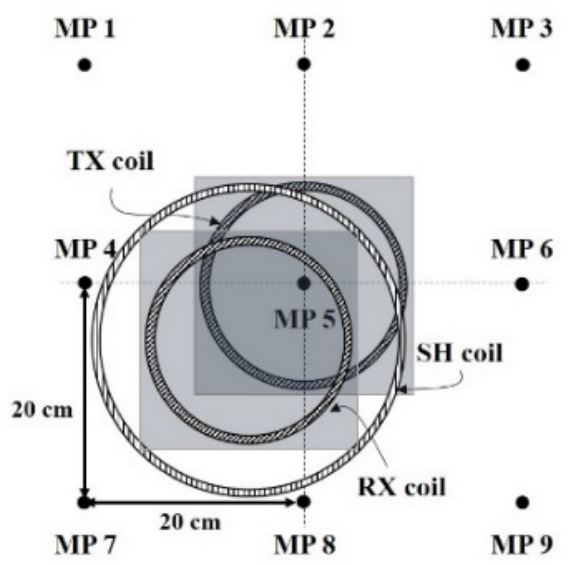

Fig. 10. EMF measurement points. 
Table 2. Current strengths and phases of the TX, RX, and SH coils

\begin{tabular}{lccccc}
\hline & $I_{T X}$ & $I_{R X}$ & $I_{S H}$ & $\Phi_{S H}$ & $P_{\text {Load }}$ \\
& $(\mathrm{A})$ & $(\mathrm{A})$ & $(\mathrm{A})$ & $\left(^{\circ}\right)$ & $(\mathrm{W})$ \\
\hline Alignment & 4.49 & 6.1 & 4.4 & -133 & 102.7 \\
$\begin{array}{c}\text { Misalignment } \\
\text { (controlled) }\end{array}$ & 4.91 & 6.1 & 5.7 & -150 & 103.9 \\
\hline
\end{tabular}

target point $p$ as the center point of the alignment condition to evenly reduce all high magnetic fields at close ranges around the system.

\section{Simulation and Experimental Results}

Fig. 11 shows the results of the simulations and experiments for the leakage magnetic field strength under alignment and misalignment conditions at nine different MPs when the $\mathrm{SH}$ coil was not applied. The magnetic field leakage increased when misalignment occurred. Under misalignment conditions, the leakage magnetic field strength around the locations where the RX coil was positioned (MPs 4, 5, 7, and 8) increased because the $I_{T X}$ had increased for transferring identical $P_{\text {Load }}$ against a decrease of $M_{\text {TXRX. }}$.

Fig. 12(a) shows the simulation and experimental results for EMFs for the $\mathrm{SH}$ system under alignment conditions. The EMFs at all MPs decreased when the SH system was applied. In particular, since point $p$ was selected as the center point for the alignment condition, the highest EMF reduction occurred at MP5, as shown in Fig. 12(a). The SP was calculated according to Eq. (15), and the average SP was the sum of the SPs from MP1-MP9 divided by $9 . B_{w o S H}$ and $B_{S H}$ were the leakage magnetic field strengths before and after the application of the $\mathrm{SH}$ coil. The average SP under the alignment condition was $67.16 \%$.

Fig. 12(b) shows the simulation and experimental results for

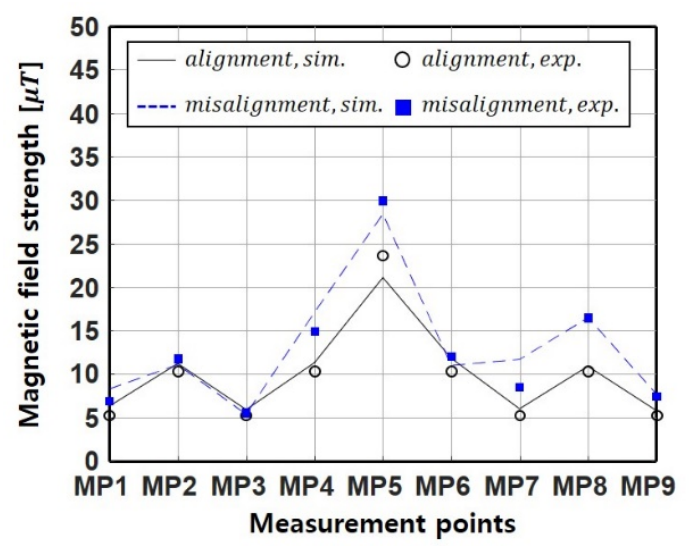

Fig. 11. EMF simulation and experimental results for different MPs under alignment and misalignment conditions of a system without a $\mathrm{SH}$ coil.

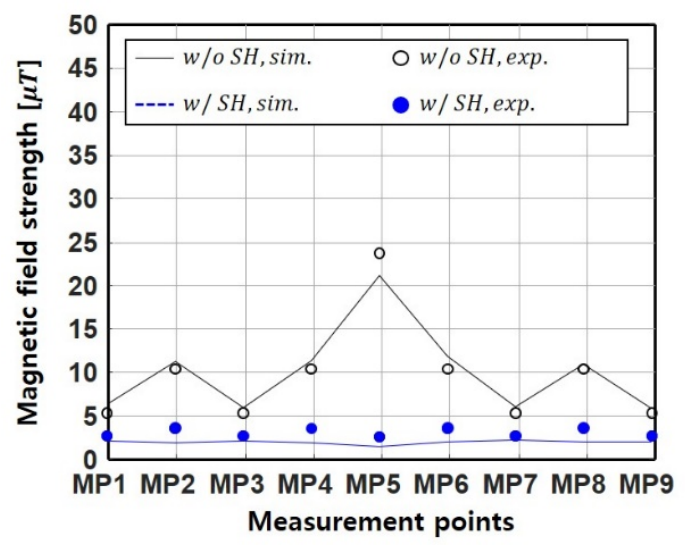

(a)

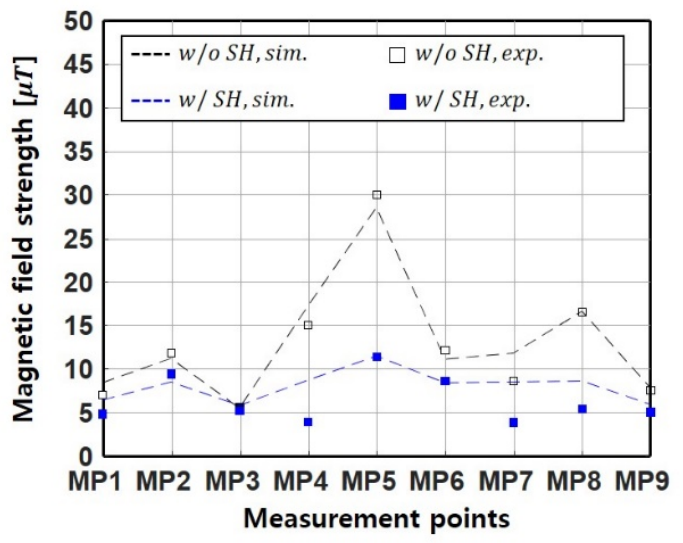

(b)

Fig. 12. EMF simulation and experimental results, with and without the $\mathrm{SH}$ coil, at different MPs under (a) alignment and (b) misalignment conditions.

EMFs according to the SH system under misalignment conditions. Although EMF leakage decreased under misalignment conditions, the average SP was 49.22\%-inferior to the alignment condition-because the current amplitude and phase of the $\mathrm{SH}$ coil were determined based on the alignment condition.

$$
S P=\left(1-\frac{B_{S H}}{B_{w o S H}}\right) \times 100(\%)
$$

Fig. 13 shows the SP variations according to the active shielding control when misalignment occurred. The SP was improved by adding active shielding control under misalignment conditions. We confirmed that this improvement in SP occurred in all MPs, as well as at the target shielding point. The average SP of the controlled SH coil was $61.74 \%$.

Consequently, the average SP under misalignment conditions was increased from $49.22 \%$ to $61.74 \%$ by applying the proposed control method. This meant that $24.6 \%$ of the EMF leakage was additionally reduced by applying active shielding control for misalignment conditions.

Table 3 shows the PTE variations under misalignment conditions. $P_{\text {in }}$ is the sum of the supplied power for TX and SH. 


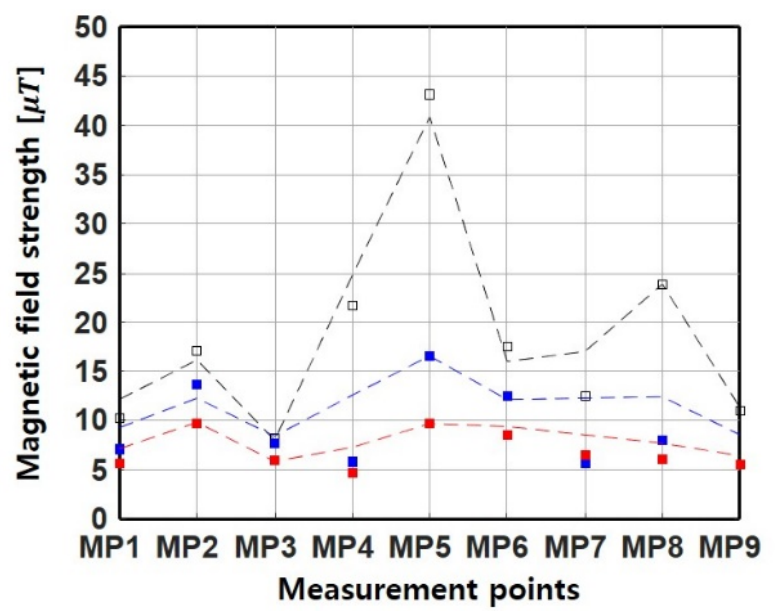

\begin{tabular}{|ll|}
\hline--- w/o SH, sim. & $\square$ w/o SH, exp. \\
$----w / S H$, sim. & $\square$ w/SH, exp. \\
$----w /$ controlled SH, sim. & $\square$ w/ controlled SH, exp. \\
\hline
\end{tabular}

Fig. 13. EMF simulation and experimental results at different MPs under misalignment conditions: without $\mathrm{SH}$ coil, with $\mathrm{SH}$ coil, and with controlled SH coil.

Table 3. PTE under misalignment conditions

\begin{tabular}{lccccc}
\hline & $\begin{array}{c}P_{\text {in }} \\
(\mathrm{W})\end{array}$ & $\begin{array}{c}V_{\text {Load }} \\
(\mathrm{V})\end{array}$ & $\begin{array}{c}I_{\text {Load }} \\
(\mathrm{A})\end{array}$ & $\begin{array}{c}P_{\text {Load }} \\
(\mathrm{W})\end{array}$ & $\begin{array}{c}\text { PTE } \\
(\%)\end{array}$ \\
\hline w/o shield & 125.44 & 31.80 & 3.23 & 102.71 & 81.88 \\
w/ shield & 143.84 & 31.65 & 3.22 & 101.91 & 70.85 \\
w/ controlled shield & 146.20 & 31.97 & 3.25 & 103.90 & 71.07 \\
\hline
\end{tabular}

The power transfer conditions for all measurements were 100 W transmission to $R_{L}$. As shown in Table 3, the proposed active shielding control method produced no additional PTE degradation, meaning that the SP could be improved without PTE degradation under misalignment conditions.

\section{CONCLUSION}

This paper proposes a control method for an independent active shielding system under misalignment conditions. Changes in the operating characteristics of WPT systems, such as $M$ and $I_{T X}$, according to the misalignment, were reflected in the shielding current control determination. Based on this, we propose a method for determining the current strength and phase of the shielding coil under misalignment conditions. When the proposed method was applied, the experimental results showed that the SP improved from $49.22 \%$ to $61.73 \%$ under misalignment conditions. Also, no further PTE reduction occurred when we applied the proposed control method. In conclusion, we verified that SP could be improved for misalignment conditions by applying independent active shielding.
This work was supported by Institute of Information \& communications Technology Planning \& Evaluation (IITP) grant funded by the Korea government (MSIT) (No. 20200-00839, Development of advanced power and signal EMC technologies for hyper-connected e-vehicle). This research was supported by Korea Electric Power Corporation (No. R20XO02-35).

\section{REFERENCES}

[1] S. D. Barman, A. W. Reza, N. Kumar, M. E. Karim, and A. B. Munir, "Wireless powering by magnetic resonant coupling: recent trends in wireless power transfer system and its applications," Renewable and Sustainable Energy Reviews, vol. 51, pp. 1525-1552, 2015.

[2] International Commission on Non-Ionizing Radiation Protection, "Guidelines for limiting exposure to timevarying electric, magnetic, and electromagnetic fields (up to $300 \mathrm{GHz}$ )," Health Physics, vol. 74, no. 4, pp. 494-522, 1998.

[3] International Commission on Non-Ionizing Radiation Protection, "Guidelines for limiting exposure to timevarying electric and magnetic fields $(1 \mathrm{~Hz}$ to $100 \mathrm{kHz}), "$ Health Physics, vol. 99, no. 6, pp. 818-836, 2010.

[4] IEEE standard for safety levels with respect to human exposure to radio frequency electromagnetic fields, $3 \mathrm{kHz}$ to $300 \mathrm{GHz}$, IEEE C95.1-2005, 2006.

[5] J. Kim, J. Kim, S. Kong, H. Kim, I. S. Suh, N. P. Suh, D. H. Cho, J. Kim, and S. Ahn, "Coil design and shielding methods for a magnetic resonant wireless power transfer system," Proceedings of the IEEE, vol. 101, no. 6, pp. 13321342, 2013.

[6] J. Kim, J. Ahn, S. Huh, K. Kim, and S. Ahn, "A coil design and control method of independent active shielding system for leakage magnetic field reduction of wireless UAV charger," IEICE Transactions on Communications, vol. 103, no. 9, pp. 889-898, 2020.

[7] S. Y. Choi, B. W. Gu, S. W. Lee, W. Y. Lee, J. Huh, and C. T. Rim, "Generalized active EMF cancel methods for wireless electric vehicles," IEEE Transactions on Power Electronics, vol. 29, no. 11, pp. 5770-5783, 2014.

[8] S. Cruciani, T. Campi, F. Maradei, and M. Feliziani, "Active shielding design for wireless power transfer systems," IEEE Transactions on Electromagnetic Compatibility, vol. 61, no. 6, pp. 1953-1960, 2019.

[9] Y. Gao, A. Ginart, K. B. Farley, and Z. T. H. Tse, "Misalignment effect on efficiency of wireless power transfer for electric vehicles," in Proceedings of 2016 IEEE Applied 
Power Electronics Conference and Exposition (APEC), Long Beach, CA, 2016, pp. 3526-3528.

[10] Y. Palagani, K. Mohanarangam, J. H. Shim, and J. R. Choi, "Wireless power transfer analysis of circular and spherical coils under misalignment conditions for biomedical implants," Biosensors and Bioelectronics, vol. 141, article no. 111283, 2019. https://doi.org/10.1016/j.bios.2019.04.051

[11] Z. Luo and X. Wei, "Mutual inductance analysis of planar coils with misalignment for wireless power transfer systems in electric vehicle," in Proceedings of 2016 IEEE Vehicle Power and Propulsion Conference (VPPC), Hangzhou,

Jangyong Ahn

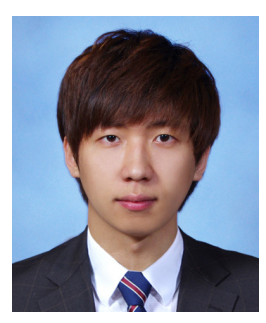

received his M.S. degree from the Cho Chun Shik Graduate School of Green Transportation at the Korea Advanced Institute of Science and Technology (KAIST), South Korea, in 2018. He is currently working toward a Ph.D. at KAIST. His research interests include power integrity in electric vehicles and $\mathrm{EMI} / \mathrm{EMC}$ and human exposure to EMF from wireless power transfer systems.

Jedok Kim

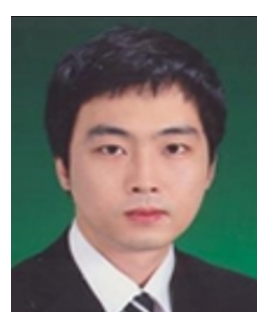

received his Ph.D. degree from the Korea Advanced Institute of Science and Technology (KAIST), Daejeon, South Korea. He is currently working on postdoctoral researcher at KAIST. His research mainly focuses on magnetic field leakage reduction and foreign object detection in wireless power transfer systems for improving human safety.

Dongryul Park

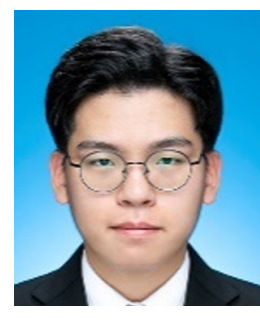

received his M.S. degree in 2021 from the Cho Chun Shik Graduate School of Green Transportation at the Korea Advanced Institute of Science and Technology (KAIST), Daejeon, South Korea, where he is currently working toward a Ph.D. His research interests include vehicular communications, electromagnetic compatibility, and optimization using deep learning.
China, 2016, pp. 1-6.

[12] S. W. Park, "Dosimetry for resonance-based wireless power transfer charging of electric vehicles," Journal of Electromagnetic Engineering and Science, vol. 15, no. 3, pp. 129133, 2015.

[13] T. Imura and Y. Hori, "Maximizing air gap and efficiency of magnetic resonant coupling for wireless power transfer using equivalent circuit and Neumann formula," IEEE Transactions on Industrial Electronics, vol. 58, no. 10, pp. 4746-4752, 2011.

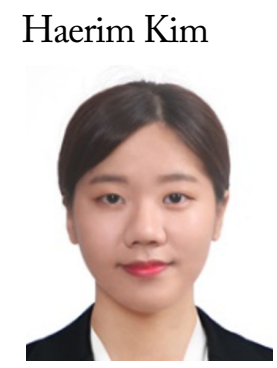

received her M.S. degree in 2019 from the Cho Chun Shik Graduate School of Green Transportation from the Korea Advanced Institute of Science and Technology (KAIST), Daejeon, South Korea, where she is currently pursuing a Ph.D. Her current research interests include wireless power transfer system design.

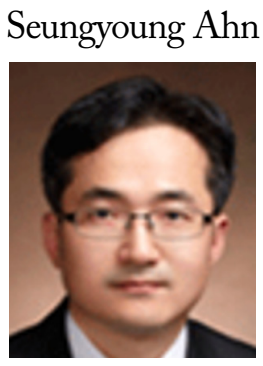

received his B.S., M.S., and Ph.D. degrees from the Korea Advanced Institute of Science and Technology (KAIST), Daejeon, South Korea, in 1998, 2000, and 2005, respectively—all in electrical engineering. From 2005 to 2009, he was a senior engineer with Samsung Electronics in Suwon, South Korea, where he was in charge of high-speed board design for laptop computer systems. He is currently an associate professor at Cho Chun Shik Graduate School of Green Transportation, KAIST. His research interests include wireless power transfer system design and electromagnetic compatibility design for electric vehicles and high-performance digital systems. 